\title{
GRÖBNER BASES AND DIMENSION FORMULAS FOR TERNARY PARTIALLY ASSOCIATIVE OPERADS
}

\author{
FATEMEH BAGHERZADEH AND MURRAY BREMNER
}

\begin{abstract}
Dotsenko and Vallette discovered an extension to nonsymmetric operads of Buchberger's algorithm for Gröbner bases of polynomial ideals. In the free nonsymmetric operad with one ternary operation $(* * *)$, we compute a Gröbner basis for the ideal generated by partial associativity $((a b c) d e)+$ $(a(b c d) e)+(a b(c d e)$. In the category of $\mathbb{Z}$-graded vector spaces with Koszul signs, the (homological) degree of $(* * *)$ may be even or odd. We use the Gröbner bases to calculate the dimension formulas for these operads.
\end{abstract}

\section{INTRODUCTION}

We consider nonsymmetric operads in the category of $\mathbb{Z}$-graded vector spaces over a field of characteristic 0 . The product is the tensor product (with Koszul signs) and the coproduct is the direct sum. Gröbner bases for operads were introduced by Dotsenko, Khoroshkin \& Vallette [5], 8]; see also [2].

Let $\mathcal{L} \mathcal{T}$ be the free nonsymmetric operad with one ternary operation $t=(* * *)$. Let $\alpha$ denote ternary partial associativity, which may be written as a tree polynomial, using partial compositions, or as a nonassociative polynomial:

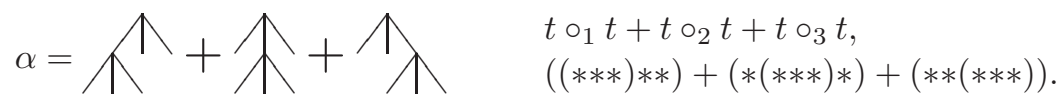

We compute a Gröbner basis for the ideal $\langle\alpha\rangle$ when $t$ has even (homological) degree so that Koszul signs are irrelevant, and when $t$ has odd degree so that Koszul signs are essential. We include details of the calculations to clarify the Gröbner basis algorithm for nonsymmetric operads. As an application, we calculate dimension formulas for the quotient operads. Similar results have been obtained independently in unpublished work of Vladimir Dotsenko.

For earlier work on partial associativity and its applications, see [1,3, 6, $9,11,13-$ 15. Recent results of Dotsenko, Shadrin \& Vallette [7] have shown that the ternary partially associative operad with an odd generator arises naturally in the homology of the poset of interval partitions into intervals of odd length and in certain De Concini-Procesi models of subspace arrangements [4] over the real numbers.

2010 Mathematics Subject Classification. Primary 18D50. Secondary 17A30, 17A40, 17A50.

Key words and phrases. Nonsymmetric operads, ternary operations, partial associativity, Gröbner bases, dimension formulas.

This research was supported by the Discovery Grant Algebraic Operads (2016) from the Natural Sciences and Engineering Research Council of Canada (NSERC). The authors thank Vladimir Dotsenko for sending us his results without proofs on Gröbner bases and dimension formulas for ternary partially associative operads (emails of $16 \& 26$ February 2018) and for helpful comments on an earlier version of this paper. 


\section{Preliminaries}

Definition 2.1. An $m$-ary tree is a rooted plane tree $p$ in which every node has no children (leaf) or $m$ children (internal node). The weight $w(p)$ counts internal nodes; the arity $\ell(p)=1+w(p)(m-1)$ counts leaves indexed $1, \ldots, \ell(p)$ from left to right. The basic tree $t$ is the $m$-ary tree of weight 1 . Set $[n]=\{1, \ldots, n\}$.

Definition 2.2. If $n \equiv 1(\bmod m-1)$ then $\mathcal{T}(n)$ is the set of $m$-ary trees of arity $n$, and $\mathcal{T}$ is the disjoint union of the $\mathcal{T}(n)$ for $n \geq 1$.

Definition 2.3. If $p, q \in \mathcal{T}$ then for $i \in[\ell(p)]$ the partial composition $p \circ_{i} q \in \mathcal{T}$ is obtained by identifying leaf $i$ of $p$ with the root of $q$.

Lemma 2.4. Starting with $t$, every $m$-ary tree of weight $w$ can be obtained by a sequence of $w-1$ partial compositions.

Lemma 2.5. Let $p, q, r$ be m-ary trees. Partial composition satisfies [2, p. 72]:

$$
\left(p \circ_{i} q\right) \circ_{j} r= \begin{cases}p \circ_{i}\left(q \circ_{j-i+1} r\right), & i \leq j \leq i+\ell(q)-1 \\ \left(p \circ_{j-\ell(q)+1} r\right) \circ_{i} q, & i+\ell(q) \leq j \leq \ell(p)+\ell(q)-1 ; \\ \left(p \circ_{j} r\right) \circ_{i+\ell(r)-1} q, & 1 \leq j \leq i-1 .\end{cases}
$$

Lemma 2.6. The set $\mathcal{T}$ with partial compositions is isomorphic to the free nonsymmetric (set) operad with one $m$-ary operation $t$.

Definition 2.7. If $n \equiv 1(\bmod m-1)$ then $\mathcal{L T}(n)$ is the vector space with basis $\mathcal{T}(n)$, and $\mathcal{L} \mathcal{T}$ is the direct sum of $\mathcal{L} \mathcal{T}(n)$ for $n \geq 1$. A tree polynomial of arity $n$ is an element of $\mathcal{L} \mathcal{T}(n)$. Partial composition in $\mathcal{T}$ extends bilinearly to $\mathcal{L} \mathcal{T}$.

Lemma 2.8. The vector space $\mathcal{L} \mathcal{T}$ with partial compositions is isomorphic to the free nonsymmetric (vector) operad with one $m$-ary operation $t$.

Definition 2.9. A relation of arity $n$ is an element of $\mathcal{L} \mathcal{T}(n) \backslash 0$. For relations $f_{1}, \ldots, f_{k}$ let $\mathcal{I}=\left\langle f_{1}, \ldots, f_{k}\right\rangle=\bigcap \mathcal{S}$ over homogeneous subspaces $\mathcal{S} \subseteq \mathcal{L} \mathcal{T}$ with $f_{1}, \ldots, f_{k} \in \mathcal{S}$, and $f \in \mathcal{S}(m), g \in \mathcal{L} \mathcal{T}(n) \Longrightarrow f \circ_{i} g, g \circ_{j} f \in \mathcal{S}(i \in[m], j \in[n])$.

The following results come from [2, $\S 3.4]$ and [8, $\S \S 2.4,3.1]$ with minor changes.

Definition 2.10. The path sequence of $p \in \mathcal{T}(n)$ is $\operatorname{path}(p)=\left(a_{1}, \ldots, a_{n}\right)$ where $a_{i}$ is the length of the path from the root to the leaf $i$.

Lemma 2.11. If $p, q \in \mathcal{T}$ then $p=q$ if and only if $\operatorname{path}(p)=\operatorname{path}(q)$.

Definition 2.12. For $p, q \in \mathcal{T}(n)$ we write $p \prec q$ and say $p$ precedes $q$ in path-lex order if and only if $\operatorname{path}(p) \prec \operatorname{path}(q)$ in lex order on $n$-tuples of positive integers. If $f \in \mathcal{L} \mathcal{T}(n)$ then its leading monomial $\ell m(f) \in \mathcal{T}(n)$ is the greatest monomial in path-lex order, and its leading coefficient $\ell c(f)$ is the coefficient of $\ell m(f)$.

Definition 2.13. If $p, q \in \mathcal{T}$ then $q$ is divisible by $p$ (written $p \mid q$ ) if $p$ is a subtree of $q$ : that is, $q=\cdots p \cdots$ where the dots denote sequences of partial compositions with parentheses. If $p \in \mathcal{T}(m), q \in \mathcal{T}(n), p \mid q$, and $f \in \mathcal{L} \mathcal{T}(m)$ then we may replace $p$ by $f$ in $q$ and use linearity and the same partial compositions to obtain

$$
M(q, p, f)=\cdots f \cdots \in \mathcal{L} \mathcal{T}(n) .
$$


Definition 2.14. If $f, g \in \mathcal{L T}$ and $\ell m(g) \mid \ell m(f)$ then the reduction of $f$ by $g$ (which eliminates the leading term of $f$ ) is

$$
R(f, g)=f-\frac{\ell c(f)}{\ell c(g)} M(\ell m(f), \ell m(g), g) .
$$

This extends to reduction of $f$ by $g_{1}, \ldots, g_{k}$; see [2, Algorithm 3.4.2.16].

Definition 2.15. If $p, q, r \in \mathcal{T}$ then $p$ is a small common multiple (SCM) of $q, r$ if $q|p, \quad r| p, \quad$ every node of $p$ is a node of $q$ or $r$ (or both), $\quad \ell(p)<\ell(q)+\ell(r)$.

Definition 2.16. If $f, g, h$ are monic tree polynomials and $\ell m(f)$ is an SCM of $\ell m(g), \ell m(h)$ then the resulting $S$-polynomial is

$$
S(f, g, h)=M(\ell m(f), \ell m(g), g)-M(\ell m(f), \ell m(h), h) .
$$

Definition 2.17. Let $G$ be a finite set of relations and let $I=\langle G\rangle$. If for all $f \in I$ there exists $g \in G$ such that $\ell m(g) \mid \ell m(f)$ then we call $G$ a Gröbner basis for $I$. We say $G$ is reduced if $\ell m(g)$ is not divisible by $\ell m(h)$ for all $g, h \in G$.

Lemma 2.18. Every operad ideal has a unique reduced Gröbner basis.

Theorem 2.19. If $I=\langle G\rangle$ then $G$ is a Gröbner basis for I if and only if for every $S C M f$ of elements $g, h \in G$ the reduction of $S(f, g, h)$ by $G$ is 0 .

\section{GRÖBNER BASES AND DIMENSION FORMULAS}

In the rest of this paper we consider a ternary operation $(m=3)$. We usually indicate the leading monomial of a tree polynomial by a bullet at the root, and write the terms of a tree polynomial from left to right in reverse path-lex order. The partially associative relation $\alpha$ corresponds to this rewrite rule:

$$
t \circ_{1} t=\mathbb{\Lambda} \longrightarrow-\widehat{N}-\widehat{\Lambda}=-t \circ_{2} t-t \circ_{3} t
$$

Theorem 3.1. For the path-lex monomial order, the following tree polynomials form the reduced Gröbner basis for $\langle\alpha\rangle$ with an operation of even degree:
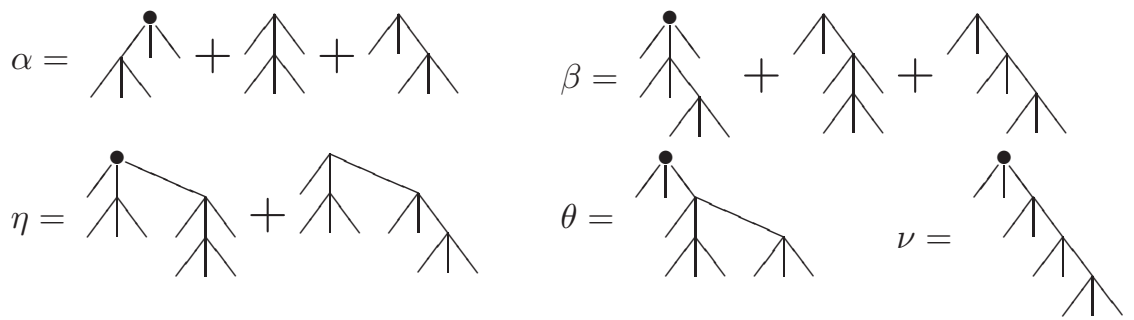

Proof. The proof consists of Lemmas 3.3 to 3.9 .

Remark 3.2. As nonassociative polynomials, the relations of Theorem 3.1 are:

$$
\begin{aligned}
& ((* * *) * *)+(*(* * *) *)+(* *(* * *)),(*(* *(* * *)) *)+(* *(*(* * *) *))+(* *(* *(* * *))), \\
& (*(* * *)(*(* * *) *))+(*(* * *)(* *(* * *))),(* *(*(* * *)(* * *))),(* *(* *(* *(* * *)))) .
\end{aligned}
$$

Lemma 3.3. There is only one $S C M$ of $\operatorname{lm}(\alpha)$ with itself; this produces reduced $S$-polynomial $\beta$, and the set $\{\alpha, \beta\}$ is self-reduced:

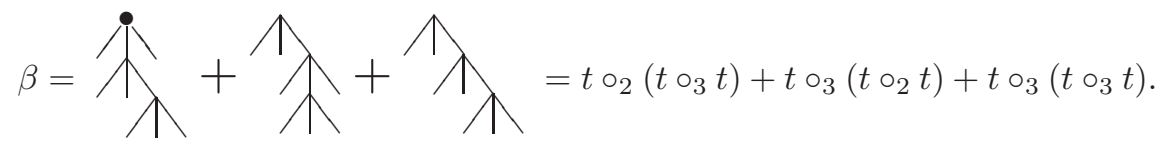


Proof. We have $\operatorname{lm}(\alpha)=t \circ_{1} t$ and hence

$$
\ell m(\alpha) \circ_{1} t=\mathbb{N}^{\mathbb{N}=t \circ_{1} \operatorname{lm}(\alpha)} .
$$

From this we obtain these tree polynomials (Definition 2.13):

$$
\alpha \circ_{1} t=\left(t \circ_{1} t\right) \circ_{1} t+\left(t \circ_{2} t\right) \circ_{1} t+\left(t \circ_{3} t\right) \circ_{1} t=t
$$

The difference is this (non-reduced) S-polynomial:

$$
\begin{aligned}
\alpha \circ_{1} t-t \circ_{1} \alpha & =\left(t \circ_{2} t\right) \circ_{1} t+\left(t \circ_{3} t\right) \circ_{1} t-t \circ_{1}\left(t \circ_{2} t\right)-t \circ_{1}\left(t \circ_{3} t\right) \\
& =\left(t \circ_{1} t\right) \circ_{4} t+\left(t \circ_{1} t\right) \circ_{5} t-\left(t \circ_{1} t\right) \circ_{2} t-\left(t \circ_{1} t\right) \circ_{3} t .
\end{aligned}
$$

We have rewritten the partial compositions (Lemma 2.5). We apply rewrite rule (2) to the top subtree $\ell m(\alpha)=t \circ_{1} t$ of each monomial (reduce using $\alpha$ ):

$$
\begin{aligned}
& -\left(t \circ_{2} t\right) \circ_{4} t-\left(t \circ_{3} t\right) \circ_{4} t-\left(t \circ_{2} t\right) \circ_{5} t-\left(t \circ_{3} t\right) \circ_{5} t \\
& +\left(t \circ_{2} t\right) \circ_{2} t+\left(t \circ_{3} t\right) \circ_{2} t+\left(t \circ_{2} t\right) \circ_{3} t+\left(t \circ_{3} t\right) \circ_{3} t .
\end{aligned}
$$

Terms 3 and 6 cancel since both monomials represent the same tree:

$$
\left(t \circ_{2} t\right) \circ_{5} t=\left(t \circ_{3} t\right) \circ_{2} t=\bigwedge
$$

Six terms remain:

$$
-\left(t \circ_{2} t\right) \circ_{4} t-\left(t \circ_{3} t\right) \circ_{4} t-\left(t \circ_{3} t\right) \circ_{5} t+\left(t \circ_{2} t\right) o_{2} t+\left(t \circ_{2} t\right) o_{3} t+\left(t \circ_{3} t\right) \circ_{3} t
$$

In terms 4 and 6 , we reduce the bottom subtree $\ell m(\alpha)=t \circ_{1} t$ using $\alpha$ :

$$
\begin{aligned}
& -t \circ_{2}\left(t \circ_{3} t\right)-t \circ_{3}\left(t \circ_{2} t\right)-t \circ_{3}\left(t \circ_{3} t\right)-t \circ_{2}\left(t \circ_{2} t\right) \\
& -t \circ_{2}\left(t \circ_{3} t\right)+t \circ_{2}\left(t \circ_{2} t\right)-t \circ_{3}\left(t \circ_{2} t\right)-t \circ_{3}\left(t \circ_{3} t\right) .
\end{aligned}
$$

Terms 4 and 6 cancel and the others combine in pairs:

$$
-2\left[t \circ_{2}\left(t \circ_{3} t\right)+t \circ_{3}\left(t \circ_{2} t\right)+t \circ_{3}\left(t \circ_{3} t\right)\right]=-2[\lambda
$$

No further reduction is possible. The monic form of the last polynomial is $\beta$. 
The relation $\beta$ corresponds to this rewrite rule:

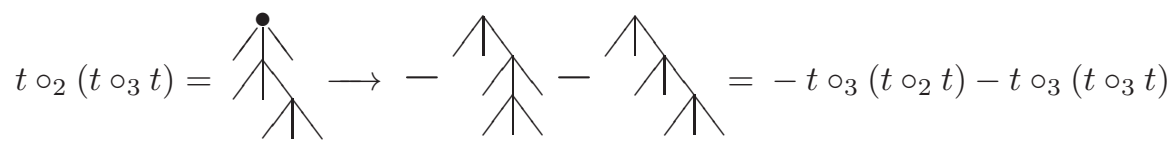

We consider separately the four SCMs of $\ell m(\alpha)=t \circ_{1} t$ and $\ell m(\beta)=t \circ_{2}\left(t \circ_{3} t\right)$.

Lemma 3.4. Identifying the second $t$ of $\ell m(\alpha)=t \circ_{1} t$ with the first $t$ of $\ell m(\beta)=$ $t \circ_{2}\left(t \circ_{3} t\right)$ produces the reduced S-polynomial $\gamma$, and $\{\alpha, \beta, \gamma\}$ is self-reduced:

$$
\gamma=2 \bigwedge_{\lambda}+\bigwedge_{\lambda}=2\left(t \circ_{3}\left(t \circ_{2} t\right)\right) \circ_{7} t+t \circ_{3}\left(t \circ_{3}\left(t \circ_{3} t\right)\right) \text {. }
$$

Proof. We have the following equations:

$$
\ell m(\alpha) \circ_{2}\left(t \circ_{3} t\right)=\left(t \circ_{1} t\right) \circ_{2}\left(t \circ_{3} t\right)=\bigwedge_{\bigwedge}=t \circ_{1}\left(t \circ_{2}\left(t \circ_{3} t\right)\right)=t \circ_{1} \ell m(\beta) .
$$

We apply the same partial compositions to $\alpha$ and $\beta$ :

$$
\begin{aligned}
\alpha \circ_{2}\left(t \circ_{3} t\right) & =\left(t \circ_{1} t\right) \circ_{2}\left(t \circ_{3} t\right)+\left(t \circ_{2} t\right) \circ_{2}\left(t \circ_{3} t\right)+\left(t \circ_{3} t\right) \circ_{2}\left(t \circ_{3} t\right), \\
t \circ_{1} \beta & =t \circ_{1}\left(t \circ_{2}\left(t \circ_{3} t\right)\right)+t \circ_{1}\left(t \circ_{3}\left(t \circ_{2} t\right)\right)+t \circ_{1}\left(t \circ_{3}\left(t \circ_{3} t\right)\right) .
\end{aligned}
$$

Taking the difference, we obtain this (non-reduced) S-polynomial:

$$
\begin{aligned}
& \left(t \circ_{1} t\right) \circ_{2}\left(t \circ_{3} t\right)+\left(t \circ_{2} t\right) \circ_{2}\left(t \circ_{3} t\right)+\left(t \circ_{3} t\right) \circ_{2}\left(t \circ_{3} t\right) \\
& -t \circ_{1}\left(t \circ_{2}\left(t \circ_{3} t\right)\right)-t \circ_{1}\left(t \circ_{3}\left(t \circ_{2} t\right)\right)-t \circ_{1}\left(t \circ_{3}\left(t \circ_{3} t\right)\right) .
\end{aligned}
$$

Terms 1 and 4 cancel, leaving

$$
\left(t \circ_{2} t\right) \circ_{2}\left(t \circ_{3} t\right)+\left(t \circ_{3} t\right) \circ_{2}\left(t \circ_{3} t\right)-t \circ_{1}\left(t \circ_{3}\left(t \circ_{2} t\right)\right)-t \circ_{1}\left(t \circ_{3}\left(t \circ_{3} t\right)\right)
$$

Terms $1,3,4$ contain the subtree $\operatorname{lm}(\alpha)=t \circ_{1} t$, so we reduce them using $\alpha$ :

$$
\begin{aligned}
& -t \circ_{2}\left(\left(t \circ_{2} t\right) \circ_{3} t\right)-t \circ_{2}\left(\left(t \circ_{3} t\right) \circ_{3} t\right)+\left(t \circ_{3} t\right) \circ_{2}\left(t \circ_{3} t\right)+\left(t \circ_{2} t\right) \circ_{3}\left(t \circ_{2} t\right) \\
& +\left(t \circ_{3} t\right) \circ_{3}\left(t \circ_{2} t\right)+\left(t \circ_{2} t\right) \circ_{3}\left(t \circ_{3} t\right)+\left(t \circ_{3} t\right) \circ_{3}\left(t \circ_{3} t\right) .
\end{aligned}
$$

We write this polynomial in terms of trees:

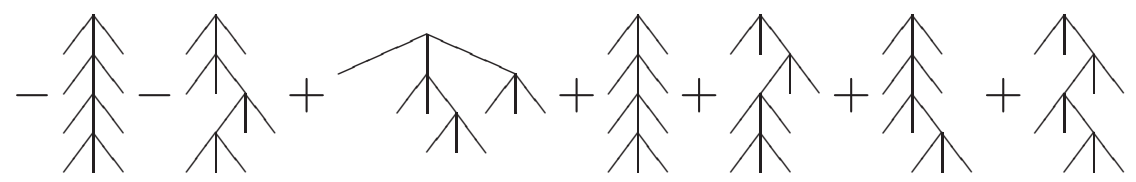


Terms 1 and 4 cancel, leaving

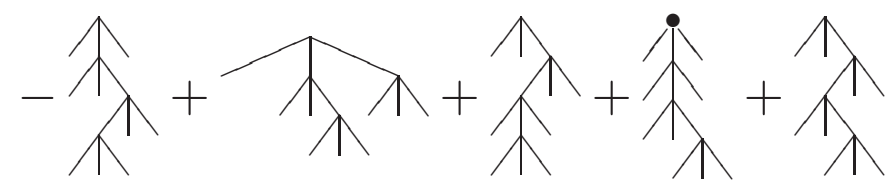

The leading monomial is divisible by $\ell m(\beta)$ but not $\ell m(\alpha)$; we reduce it using $\beta$ :

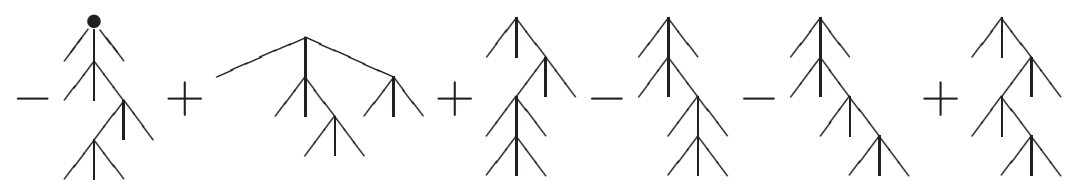

The leading monomial is divisible by $\alpha$ (bottom) and $\beta$ (top). Using $\alpha$ gives

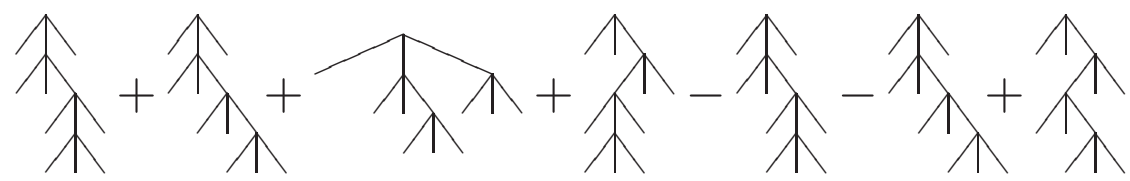

Terms 1, 5 and terms 2, 6 cancel, leaving

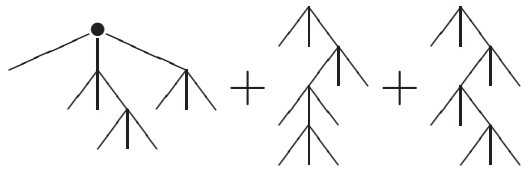

We reduce the leading monomial using $\beta$ :

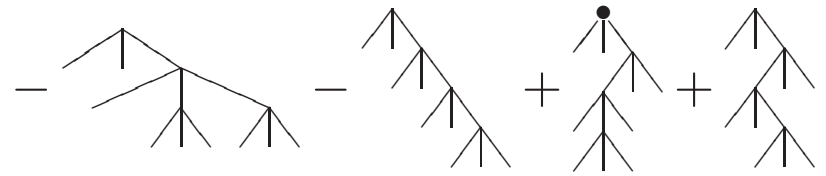

Terms 1, 2 cannot be reduced; terms 3,4 can be reduced by $\alpha$ :

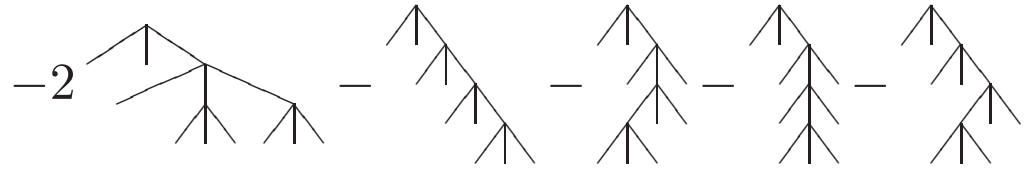

We reduce terms 3,5 by $\alpha$ :

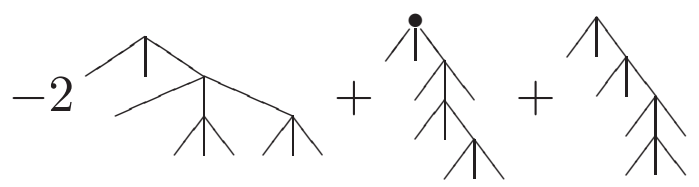

If we reduce term 2 using $\beta$ then two terms cancel and we obtain $-\gamma$.

Lemma 3.5. Identifying the first $t$ of $\ell m(\alpha)=t \circ_{1} t$ and the first $t$ of $\ell m(\beta)=$ $t \circ_{2}\left(t \circ_{3} t\right)$ we obtain the S-polynomial $\delta$, and $\{\alpha, \beta, \delta\}$ is self-reduced:

$$
\left.\left.\delta=\bigwedge_{\lambda}=\left(t \circ_{3}\left(t \circ_{2} t\right)\right) \circ_{2} t\right)+\left(t \circ_{3}\left(t \circ_{3} t\right)\right) \circ_{2} t\right)+\left(t \circ_{3}\left(t \circ_{3}\left(t \circ_{3} t\right)\right)\right. \text {. }
$$


Proof. We have the equations

$\ell m(\alpha) \circ_{4}\left(t \circ_{3} t\right)=\left(t \circ_{1} t\right) \circ_{4}\left(t \circ_{3} t\right)=\bigwedge$

We apply the same partial compositions to $\alpha$ and $\beta$ :

$$
\begin{aligned}
\alpha \circ_{4}\left(t \circ_{3} t\right) & =\left(t \circ_{1} t\right) \circ_{4}\left(t \circ_{3} t\right)+\left(t \circ_{2} t\right) \circ_{4}\left(t \circ_{3} t\right)+\left(t \circ_{3} t\right) \circ_{4}\left(t \circ_{3} t\right), \\
\beta \circ_{1} t & =\left(t \circ_{2}\left(t \circ_{3} t\right)\right) \circ_{1} t+\left(t \circ_{3}\left(t \circ_{2} t\right)\right) \circ_{1} t+\left(t \circ_{3}\left(t \circ_{3} t\right)\right) \circ_{1} t .
\end{aligned}
$$

The resulting S-polynomial is

$$
\begin{aligned}
& \left(t \circ_{1} t\right) \circ_{4}\left(t \circ_{3} t\right)+\left(t \circ_{2} t\right) \circ_{4}\left(t \circ_{3} t\right)+\left(t \circ_{3} t\right) \circ_{4}\left(t \circ_{3} t\right) \\
& -\left(t \circ_{2}\left(t \circ_{3} t\right)\right) \circ_{1} t-\left(t \circ_{3}\left(t \circ_{2} t\right)\right) \circ_{1} t-\left(t \circ_{3}\left(t \circ_{3} t\right)\right) \circ_{1} t .
\end{aligned}
$$

Terms 1, 4 cancel, leaving

$$
\left(t \circ_{2} t\right) \circ_{4}\left(t \circ_{3} t\right)+\left(t \circ_{3} t\right) o_{4}\left(t \circ_{3} t\right)-\left(t \circ_{3}\left(t \circ_{2} t\right)\right) o_{1} t-\left(t \circ_{3}\left(t \circ_{3} t\right)\right) o_{1} t
$$

We reduce terms 3,4 using $\alpha$ :

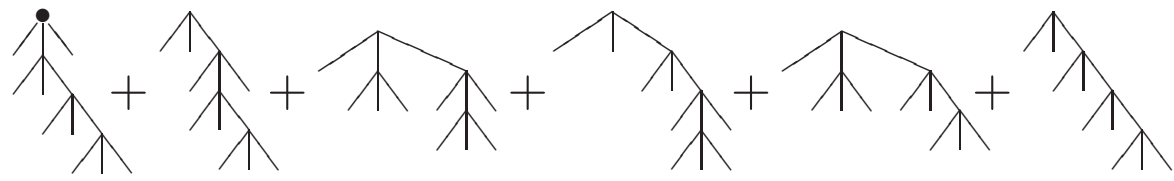

Reducing term 1 using $\beta$ gives
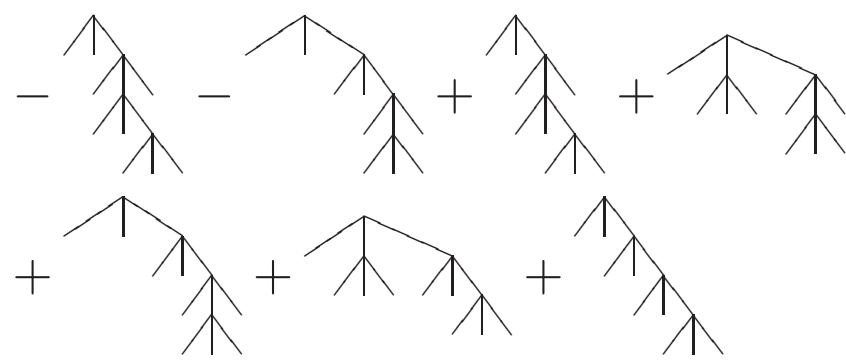

Terms 1, 3 and terms 2, 5 cancel; no further reduction is possible, producing $\delta$.

Lemma 3.6. Identifying the first $t$ of $\ell m(\alpha)=t \circ_{1} t$ with the second $t$ of $\ell m(\beta)=$ $t \circ_{2}\left(t \circ_{3} t\right)$ we obtain the S-polynomial $\epsilon$ and $\{\alpha, \beta, \epsilon\}$ is self-reduced:

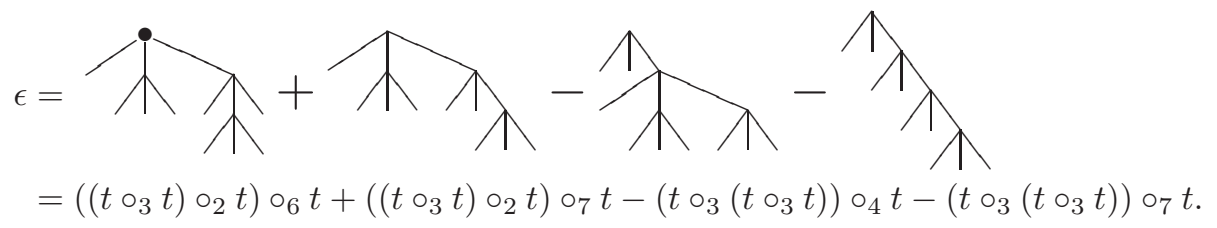

Proof. We have the equations

$t \circ_{2}\left(\ell m(\alpha) \circ_{5} t\right)=t \circ_{2}\left(\left(t \circ_{1} t\right) \circ_{5} t\right)=\left(t \circ_{2}\left(t \circ_{3} t\right)\right) \circ_{2} t=\ell m(\beta) \circ_{2} t$. 
The resulting S-polynomial $t \circ_{2}\left(\alpha \circ_{5} t\right)-\beta \circ_{2} t$ is

$$
\begin{aligned}
& t \circ_{2}\left(\left(t \circ_{1} t\right) \circ_{5} t\right)+t \circ_{2}\left(\left(t \circ_{2} t\right) \circ_{5} t\right)+t \circ_{2}\left(\left(t \circ_{3} t\right) \circ_{5} t\right) \\
& -\left(t \circ_{2}\left(t \circ_{3} t\right)\right) \circ_{2} t-\left(t \circ_{3}\left(t \circ_{2} t\right)\right) \circ_{2} t-\left(t \circ_{3}\left(t \circ_{3} t\right)\right) \circ_{2} t .
\end{aligned}
$$

Terms 1, 4 cancel, leaving

$$
t \circ_{2}\left(\left(t \circ_{2} t\right) \circ_{5} t\right)+t \circ_{2}\left(\left(t \circ_{3} t\right) \circ_{5} t\right)-\left(t \circ_{3}\left(t \circ_{2} t\right)\right) \circ_{2} t-\left(t \circ_{3}\left(t \circ_{3} t\right)\right) \circ_{2} t
$$

We reduce terms 1,2 using $\beta$ :

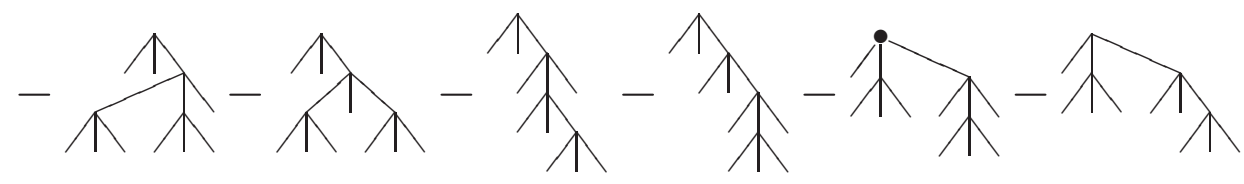

Reducing terms 1, 2 using $\alpha$ gives

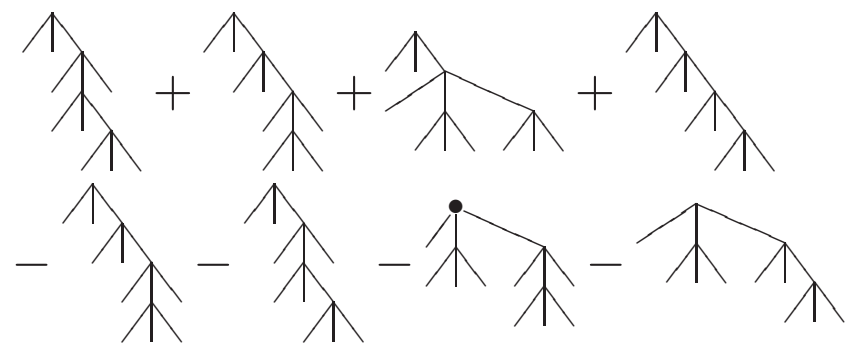

Terms 1, 6 and terms 2, 5 cancel. No further reduction is possible, giving $-\epsilon$.

Lemma 3.7. Identifying the first $t$ of $\ell m(\alpha)=t \circ_{1} t$ with the third $t$ of $\ell m(\beta)=$ $t \circ_{2}\left(t \circ_{3} t\right)$ we obtain new $S$-polynomial $\zeta$, and $\{\alpha, \beta, \zeta\}$ is self-reduced:

$$
\zeta=\bigwedge_{\lambda} \bigwedge_{\lambda}=t \circ_{3}\left(\left(t \circ_{2} t\right) \circ_{5} t\right)-t \circ_{3}\left(t \circ_{3}\left(t \circ_{3} t\right)\right) \text {. }
$$

Proof. We have the equations

$$
\left(t \circ_{2} t\right) \circ_{4} \operatorname{lm}(\alpha)=\left(t \circ_{2} t\right) \circ_{4}\left(t \circ_{1} t\right)=\bigwedge_{\lambda}=\left(t \circ_{2}\left(t \circ_{3} t\right)\right) \circ_{4} t=\ell m(\beta) \circ_{4} t .
$$

The resulting S-polynomial $\left(t \circ_{2} t\right) \circ_{4} \alpha-\beta \circ_{4} t$ is

$$
\begin{aligned}
& \left(t \circ_{2} t\right) \circ_{4}\left(t \circ_{1} t\right)+\left(t \circ_{2} t\right) \circ_{4}\left(t \circ_{2} t\right)+\left(t \circ_{2} t\right) \circ_{4}\left(t \circ_{3} t\right) \\
& -\left(t \circ_{2}\left(t \circ_{3} t\right)\right) \circ_{4} t-\left(t \circ_{3}\left(t \circ_{2} t\right)\right) \circ_{4} t-\left(t \circ_{3}\left(t \circ_{3} t\right)\right) \circ_{4} t .
\end{aligned}
$$

Terms 1, 4 cancel, leaving

$$
\left(t \circ_{2} t\right) \circ_{4}\left(t \circ_{2} t\right)+\left(t \circ_{2} t\right) \circ_{4}\left(t \circ_{3} t\right)-\left(t \circ_{3}\left(t \circ_{2} t\right)\right) \circ_{4} t-\left(t \circ_{3}\left(t \circ_{3} t\right)\right) \circ_{4} t
$$




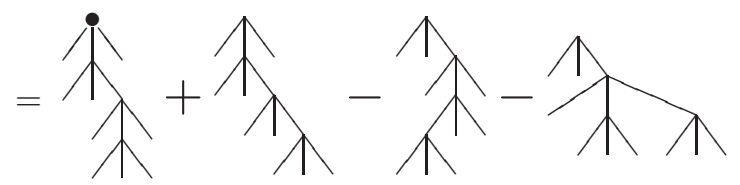

We use $\beta$ to reduce terms 1,2 :

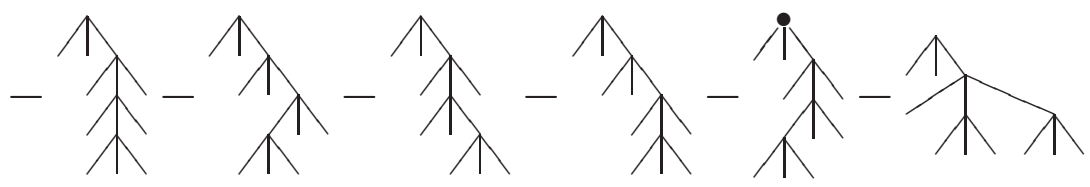

We use $\alpha$ to reduce terms 2, 5:

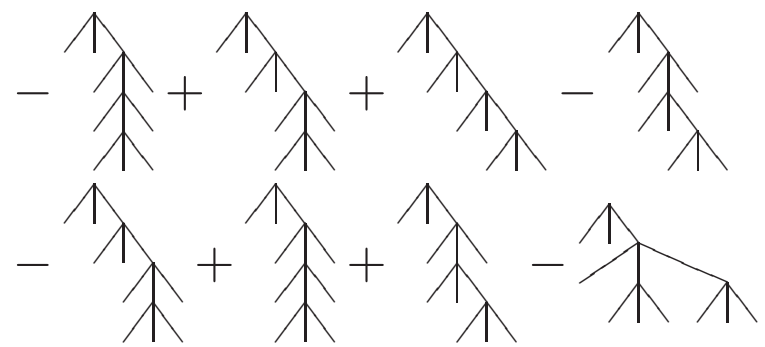

Terms 1, 6 and 2, 5 and 4, 7 cancel. No further reduction is possible, giving $-\zeta$.

Lemma 3.8. The polynomials $\gamma, \delta, \epsilon, \zeta$ span a subspace with basis $\eta, \theta, \nu$.

Proof. It is easy to see that

$$
\eta=\frac{1}{3}(\gamma+\delta+2 \epsilon), \quad \theta=\frac{1}{3}(2 \gamma-\delta+\epsilon), \quad \nu=-\frac{1}{3}(\gamma-2 \delta+2 \epsilon),
$$

and that these three polynomials form a basis of $\operatorname{span}(\gamma, \delta, \epsilon, \zeta)$.

Lemma 3.9. Every S-polynomial obtained from $\alpha, \beta, \eta, \theta, \nu$ reduces to 0.

Proof. If either $f$ or $g$ is a monomial then clearly every S-polynomial obtained from $f$ and $g$ reduces to 0 . We have already considered S-polynomials from $\alpha$ and $\beta$; the other cases are $\alpha, \eta$ and $\beta, \eta$ and $\eta, \eta$ with many subcases. We give details for the most difficult subcase and leave the others as exercises. These calculations can be simplified using the triangle lemma for nonsymmetric operads [2, Prop. 3.5.3.2].

We identify the second $t$ of $\ell m(\alpha)$ with the first $t$ of $\ell m(\eta)$ and obtain this SCM:

$\ell m(\alpha)=t \circ_{1} t, \quad \ell m(\eta)=\left(t \circ_{2} t\right) \circ_{5}\left(t \circ_{2} t\right), \quad\left(\ell m(\alpha) \circ_{2} t\right) \circ_{5}\left(t \circ_{2} t\right)=t \circ_{1} \ell m(\eta)$.

To save space we switch to nonassociative notation. We obtain the S-polynomial

$$
\begin{aligned}
& \left(\alpha \circ_{2} t\right) \circ_{5}\left(t \circ_{2} t\right)-t \circ_{1} \eta= \\
& (*((* * *)(*(* * *) *) *) *)+(*(* * *)((*(* * *) *) * *))-((*(* * *)(* *(* * *)) * *) .
\end{aligned}
$$

Rewrite rules (2) and (3) have this form; the letters represent submonomials:

$$
\begin{aligned}
((v w x) y z) & \longmapsto-(v(w x y) z)-(v w(x y z)), \\
(t(u v(w x y)) z) & \longmapsto-(t u(v(w x y) z))-(t u(v w(x y z))) .
\end{aligned}
$$

When we apply (4) or (5) we use bars to indicate the submonomials. To begin we reduce all three monomials in the S-polynomial using $\alpha$ and obtain

$$
(*((\bar{*} \bar{*} \bar{*}) \overline{(*(* * *) *)} \bar{*}) *)+(*(* * *)((\overline{*(* * *)} \bar{*}) \bar{*} \bar{*}))-((\overline{*(* * *)} \overline{(* *(* * *))} \bar{*} \bar{*})=
$$




$$
\begin{aligned}
& -(*(*(* *(*(* * *) *)) *) *)-(*(* *(*(*(* * *) *) *)) *)-(*(* * *)(*((\bar{*} \bar{*} \bar{*}) \bar{*} \bar{*}) *)) \\
& -(*(* * *)(*(* * *)(* * *)))+(*((\bar{*} \bar{*} \overline{(* *(* * *))} \bar{*}) *)+(*(* * *)((\bar{*} \overline{(* * *)}) \bar{*} \bar{*})) .
\end{aligned}
$$

Terms 3, 5, 6 reduce using $\alpha$ as indicated; term 4 is $\theta \circ_{2} t$ and reduces to 0 :

$$
\begin{aligned}
& -(*(\bar{*}(\bar{*} \bar{*}(\bar{*}(\overline{* *}) \bar{*}) \bar{*}) *)-(\bar{*}(\bar{*} \bar{*}(\bar{*}(\overline{*(* * *)}) \bar{*})) \bar{*})+(*(* * *)(*(*(* * *) *) *)) \\
& +(*(* * *)(\bar{*}(\bar{*} \bar{*}(\bar{*} \bar{*})) \bar{*}))-(*(\bar{*}(\bar{*} \bar{*}(\bar{*} \bar{*}(\overline{* *})) \bar{*}) *)-(\bar{*}(\bar{*} \bar{*}(\bar{*}(\bar{*}(* * *)) \bar{*}) \bar{*}) \\
& -(*(* * *)(*(*(* * *) *) *))-(*(* * *)(* *(* * *) *))) .
\end{aligned}
$$

Terms 3, 7 cancel, and terms 1, 2, 4, 5, 6 reduce using $\beta$ as indicated:

$$
\begin{aligned}
& (\bar{*}(\bar{*} \bar{*}(\bar{*}(\overline{*(* * *) *}) \bar{*}) \bar{*})+(\bar{*}(\bar{*} \bar{*}(\bar{*} \bar{*}(\overline{(* * *) * *})) \bar{*})+(* *(*(*(*(* * *) *)) *)) \\
& +(* *(* *((*(* * *) *) * *))-(*(* * *)(* *(*(* * *) *)))-(*(* * *)(* *(* *(* * *)))) \\
& +(\bar{*}(\bar{*} \bar{*}(\bar{*}(\overline{* *(* * *)}) \bar{*})) \bar{*})+(\bar{*}(\bar{*} \bar{*}(\bar{*} \bar{*}(\overline{*(* * *) *)}) \bar{*})+(* *(*(*(* *(* * *)) *)) *) \\
& +(* *(* *((* *(* * *)) * *)))-(*(* * *)(* *((* * *) * *))) .
\end{aligned}
$$

Terms 1, 2, 7, 8 reduce using $\beta$ as indicated; term 6 is $\nu \circ_{2} t$ and reduces to 0 ; omitting terms which cancel, we obtain

$$
\begin{aligned}
& (* *(*(* *((\bar{*} \bar{*} \bar{*}) \bar{*} \bar{*})) *))+(* *(* *(*((\bar{*} \bar{*} \bar{*}) \bar{*} \bar{*}) *)))-(*(* * *)(* *(*(* * *) *))) \\
& -(* *(*(*(* *(* * *)) *) *))-(* *(*(* *(*(* * *) *)) *))-(* *(* *(*(*(* * *)) *) *)) \\
& +(* *(* *((* *(* * *)) * *)))-(*(* * *)(* *((\bar{*} \bar{*} \bar{*}) \bar{*} \bar{*}))) .
\end{aligned}
$$

Terms 1, 2, 8 reduce using $\alpha$ as indicated; omitting terms which cancel, we obtain:

$$
\begin{aligned}
& -2(* *(\bar{*}(\bar{*} \bar{*}(\bar{*}(\overline{* * *}) \bar{*})) \bar{*}))-2(* *(* *(*(*(* * *) *) *)))-(* *(\bar{*}(\bar{*} \bar{*}(\bar{*} \bar{*}(\overline{* * *}))) \bar{*})) \\
& -(* *(* *(\bar{*}(\bar{*} \bar{*}(\bar{*} \bar{*} \bar{*})) \bar{*}))) .
\end{aligned}
$$

Terms 1, 3, 4 reduce using $\beta$ as indicated. Some terms cancel, and others reduce to 0 using $\nu$, leaving the single term $(* *(* *(\bar{*}(\bar{*} \bar{*}(\bar{*} \bar{*} \bar{*})) \bar{*})))$. We reduce using $\beta$ and then both terms reduce to 0 using $\nu$. This completes the proof of Theorem 3.1 .

We use Theorem 3.1 to calculate the dimensions of the homogeneous components of the ternary partially associative operad $\mathcal{T} \mathcal{P} \mathcal{A}=\mathcal{L} \mathcal{T} /\langle\alpha\rangle$ with an operation of even (homological) degree. Theorem 3.11 below implies the conditional result of Goze \& Remm [11, Theorem 15]; our proof using Gröbner bases is much simpler. For a more general conjecture, see [2, Conjecture 10.3.2.6, case 6].

Lemma 3.10. For $n=1,3,5,7$ we have

$$
\operatorname{dim} \mathcal{T} \mathcal{P} \mathcal{A}(1)=\operatorname{dim} \mathcal{T} \mathcal{P} \mathcal{A}(3)=1, \quad \operatorname{dim} \mathcal{T} \mathcal{P} \mathcal{A}(5)=2, \quad \operatorname{dim} \mathcal{T} \mathcal{P} \mathcal{A}(7)=4
$$

For $\mathcal{T} \mathcal{P} \mathcal{A}(5)$ a monomial basis in increasing path-lex order is

$$
T_{1}=\bigwedge, T_{2}=\bigwedge
$$

For $\mathcal{T} \mathcal{P} \mathcal{A}(7)$ a monomial basis in increasing path-lex order is

$$
T_{3}=\bigwedge_{\bigwedge}, T_{4}=\bigwedge_{\bigwedge}, T_{5}=\bigwedge_{\lambda}, T_{6}=\bigwedge
$$


Proof. The case $n=1$ is trivial, and for $n=3$ we have only the basic tree $t$. For $n=5$, the monomial $t \circ_{1} t$ reduces by $\alpha$, leaving only $T_{1}=t \circ_{2} t$ and $T_{2}=t \circ_{3} t$. For $n=7$, we have (i) $T_{1} \circ_{i} t$ : if $i=1,3$ the result reduces by $\alpha$, and if $i=2,4,5$ we obtain $T_{5}, T_{4}, T_{3}$; (ii) $t \circ_{i} T_{1}$ : if $i=1,2$ the result reduces by $\alpha, \beta$, and if $i=3$ we obtain $T_{3}$; (iii) $T_{2} \circ_{i} t$ : if $i=1,2$ the result reduces by $\alpha$, if $i=3$ we obtain $T_{6}$, if $i=4$ the result reduces by $\beta$, and if $i=5$ we obtain $T_{5}$; (iv) $t \circ_{i} T_{2}$ : if $i=1$ the result reduces by $\alpha$, if $i=2,3$ we obtain $T_{6}, T_{4}$. Clearly $T_{3}, \ldots, T_{6}$ cannot be reduced using $\alpha$ or $\beta$, which proves linear independence.

Theorem 3.11. For weight $k \geq 3$ we have $\operatorname{dim} \mathcal{T} \mathcal{P} \mathcal{A}(2 k+1)=k+1$.

Proof. Let $M_{0}$ be the tree with only one vertex, set $M_{1}=t$, and for $\ell \geq 2$ define

$$
M_{\ell}=t \circ_{2}\left(t \circ_{2}\left(t \circ_{2} \cdots\left(t \circ_{2} t\right) \cdots\right)\right) \quad(\ell \text { copies of } t) .
$$

Consider $k+1$ monomials of weight $k$ in increasing path-lex order where $\ell=M_{\ell}$ :

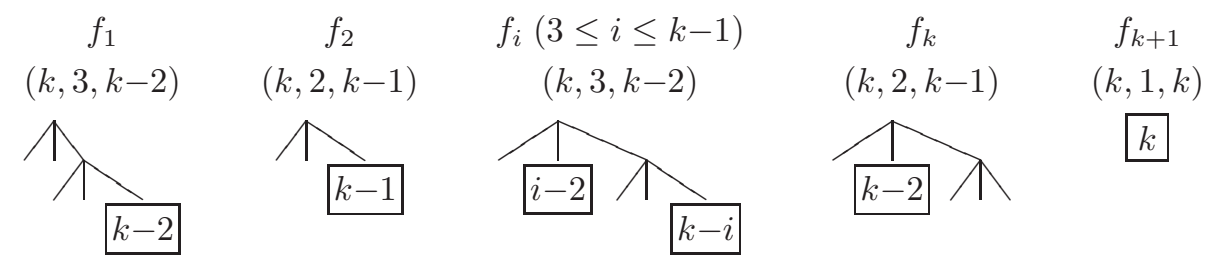

We say a leaf is left (middle, right) if it is the left (middle, right) child of its parent. The ordered triples above give the number of left (middle, right) leaves. We have

$f_{1}=t \circ_{3}\left(t \circ_{3} M_{k-2}\right), \quad f_{2}=t \circ_{3} M_{k-1}, \quad f_{i}=\left(t \circ_{3}\left(t \circ_{3} M_{k-i}\right)\right) \circ_{2} M_{i-2}(3 \leq i \leq k)$.

For $3 \leq i \leq k-1$, we obtain $f_{i+1}$ from $f_{i}$ by moving the bottom $t$ of the right-right subtree to the middle subtree. We will show that $f_{1}, \ldots, f_{k+1}$ form a basis of $\mathcal{T P A}(2 k+1)$. For linear independence, we simply observe that no $f_{i}(1 \leq i \leq k)$ can be reduced using any Gröbner basis element $\alpha, \beta, \eta, \theta, \nu$.

To prove that $f_{1}, \ldots, f_{k+1}$ span $\mathcal{T} \mathcal{P} \mathcal{A}(2 k+1)$ we use induction on $k \geq 3$. Basis: Lemma 3.10 gives $f_{1}=T_{3}, f_{2}=T_{4}, f_{3}=T_{5}, f_{4}=T_{6}$. Induction: Assume that $f_{1}, \ldots, f_{k+1}$ span $\mathcal{T} \mathcal{P} \mathcal{A}(2 k+1)$ and write $f_{1}^{\prime}, \ldots, f_{k+2}^{\prime}$ for the monomials of weight $k+1$. For each $f_{i}$ in $\mathcal{T P} \mathcal{A}(2 k+1)$ we obtain monomials of weight $k+1$ in two ways:

(1) $t \circ_{j} f_{i}$ for $j \in[3], i \in[k+1]$;

(2) $f_{i} \circ_{j} t$ for $i \in[k+1], j \in[2 k+1]$.

Case 1: If $j=1$ then $t \circ_{1} f_{i}$ reduces by $\alpha$. If $j=2$ then $t \circ_{2} f_{i}$ reduces by $\beta$ for $i \in[k]$, and $t \circ_{2} f_{k+1}=M_{k+1}=f_{k+2}^{\prime}$. If $j=3$ then $t \circ_{3} f_{1}$ reduces using $\nu$, $t \circ_{3} f_{2}=f_{1}^{\prime}, t \circ_{3} f_{i}$ reduces using $\theta$ for $i \in[k]$, and $t \circ_{3} f_{k+1}=f_{2}^{\prime}$.

Case 2 has three subcases depending on where we attach $t$. If we attach to a left leaf of $f_{i}$ then the result reduces by $\alpha$. If we attach to a right leaf then for $f_{1}$ the result reduces by $\nu$ or $\beta$, for $f_{2}, \ldots, f_{k}$ the result reduces by $\beta$ or $\theta$, and for $f_{k+1}$ either we obtain $f_{k+1}^{\prime}$ or the result reduces by $\beta$. If we attach to a middle leaf of $f_{1}$ then we obtain either $f_{3}^{\prime}$ or $f_{1}^{\prime}$ or the result reduces by $\theta$. If we attach to a middle leaf of $f_{2}$ then we obtain either $f_{3}^{\prime}$ or $f_{2}^{\prime}$. If we attach to a middle leaf of $f_{i}$ for $3 \leq i \leq k$ then we obtain $f_{j}^{\prime}$ for $3 \leq j \leq k+1$ or the result reduces by $\theta$. If we attach to the middle leaf of $f_{k+1}$ then we obtain $f_{k+2}^{\prime}$.

We now assume that the ternary operation $t$ has odd (homological) degree. Thus every tree has even or odd parity depending the number of internal nodes. We write 
$|f| \in\{0,1\}$ for the parity of $f$. We must include Koszul signs in the relations for partial compositions: transposing two odd elements introduces a minus sign.

Lemma 3.12. [12, Def. 1.1] If $p, q, r \in \mathcal{T}$ then

$$
\left(p \circ_{i} q\right) \circ_{j} r= \begin{cases}p \circ_{i}\left(q \circ_{j-i+1} r\right) & i \leq j \leq i+\ell(q)-1 \\ (-1)^{|q||r|}\left(p \circ_{j-\ell(q)+1} r\right) \circ_{i} q & i+\ell(q) \leq j \leq \ell(p)+\ell(q)-1 \\ (-1)^{|q||r|}\left(p \circ_{j} r\right) \circ_{i+\ell(r)-1} q & 1 \leq j \leq i-1\end{cases}
$$

Theorem 3.13. The relation $\alpha$ is a Gröbner basis for $\langle\alpha\rangle$ in the free nonsymmetric operad with a ternary operation of odd homological degree.

Proof. The first few steps are identical to those for an even operation. The leading monomial $\ell m(\alpha)=t \circ_{1} t$ overlaps with itself in one way to produce this SCM:

$$
\mathbb{N}^{\mathbb{N}}=\ell m(\alpha) \circ_{1} t=t \circ_{1} \ell m(\alpha) .
$$

We apply the same partial compositions to $\alpha$ instead of $\ell m(\alpha)$ :

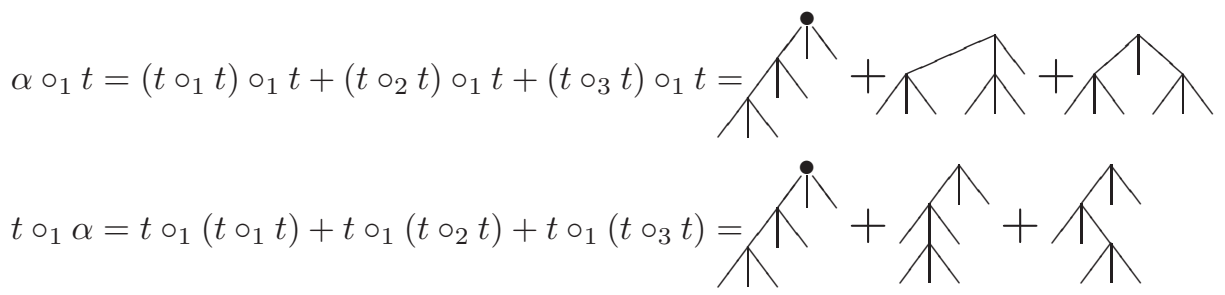

The difference is this (non-reduced) S-polynomial:

$$
\alpha \circ_{1} t-t \circ_{1} \alpha=\left(t \circ_{2} t\right) \circ_{1} t+\left(t \circ_{3} t\right) \circ_{1} t-t \circ_{1}\left(t \circ_{2} t\right)-t \circ_{1}\left(t \circ_{3} t\right)=
$$

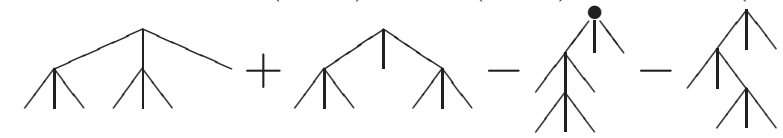

Lemma 3.12 (case 3 ), $p=q=r=t$, with $i, j=2,1$ and $i, j=3,1$ gives

$$
\left(t \circ_{2} t\right) \circ_{1} t=-\left(t \circ_{1} t\right) \circ_{4} t, \quad\left(t \circ_{3} t\right) \circ_{1} t=-\left(t \circ_{1} t\right) \circ_{5} t .
$$

Lemma 3.12 (case 1 ), $p=q=r=t$, with $i, j=2,2$ and $i, j=1,3$ gives

$$
-t \circ_{1}\left(t \circ_{2} t\right)=-\left(t \circ_{1} t\right) \circ_{2} t . \quad-t \circ_{1}\left(t \circ_{3} t\right)=-\left(t \circ_{1} t\right) \circ_{3} t .
$$

Therefore (6) equals

$$
-\left(t \circ_{1} t\right) \circ_{4} t-\left(t \circ_{1} t\right) \circ_{5} t-\left(t \circ_{1} t\right) \circ_{2} t-\left(t \circ_{1} t\right) \circ_{3} t .
$$

We reduce each monomial using $\alpha$ and obtain

$$
\begin{aligned}
& \left(t \circ_{2} t\right) \circ_{4} t+\left(t \circ_{3} t\right) \circ_{4} t+\left(t \circ_{2} t\right) \circ_{5} t+\left(t \circ_{3} t\right) \circ_{5} t \\
& +\left(t \circ_{2} t\right) \circ_{2} t+\left(t \circ_{3} t\right) \circ_{2} t+\left(t \circ_{2} t\right) \circ_{3} t+\left(t \circ_{3} t\right) \circ_{3} t .
\end{aligned}
$$

Terms 3, 6 cancel by Lemma 3.12 (case 2), $\left(t \circ_{2} t\right) \circ_{5} t=-\left(t \circ_{3} t\right) \circ_{2} t$, leaving

$\left(t \circ_{2} t\right) \circ_{4} t+\left(t \circ_{3} t\right) \circ_{4} t+\left(t \circ_{3} t\right) \circ_{5} t+\left(t \circ_{2} t\right) \circ_{2} t+\left(t \circ_{2} t\right) \circ_{3} t+\left(t \circ_{3} t\right) \circ_{3} t=$

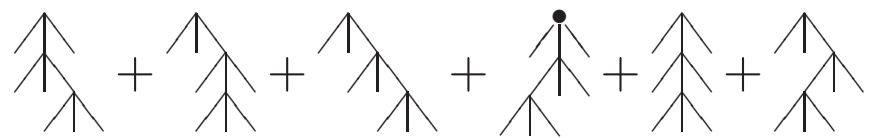

We reduce terms 4, 6 using $\alpha$; this cancels terms 1,5 and terms 2,3 . 
Theorem 3.14. For an odd operation, the dimension of the ternary partially associative operad the binary Catalan number (in the weight grading).

Proof. Relation $\alpha$ shows that any left subtree reduces, so the dimension for weight $w$ is the number of binary trees of weight $w$.

\section{REFERENCES}

[1] M. R. Bremner: On free partially associative triple systems. Comm. Algebra 28, 4 (2000) 2131-2145.

[2] M. R. Bremner, V. Dotsenko: Algebraic Operads: An Algorithmic Companion. Chapman and Hall/CRC, Boca Raton, 2016.

[3] F. Chapoton: Sur une opérade ternaire liée aux treillis de Tamari. Ann. Fac. Sci. Toulouse Math. (6) 20, 4 (2011) 843-869.

[4] C. De Concini, C. Procesi: Wonderful models of subspace arrangements. Selecta Math. (N.S.) 1 (1995), no. 3, 459-494

[5] V. Dotsenko, A. Khoroshkin: Gröbner bases for operads. Duke Math. J. 153 (2010), no. 2, 363-396.

[6] V. Dotsenko, M. Markl, E. Remm: Non-Koszulness of operads and positivity of Poincaré series. arXiv:1604.08580 [math.KT] (submitted 28 April 2016).

[7] V. Dotsenko, S. Shadrin, B. Vallette: Noncommutative $\bar{M}_{0, n+1}$. arxiv.org/abs/1510.03261 (submitted on 12 October 2015).

[8] V. Dotsenko, B. Vallette: Higher Koszul duality for associative algebras. Glasg. Math. J. 55, A (2013) 55-74.

[9] A. V. Gnedbaye: Les algèbres $k$-aires et leurs opérades. C. R. Acad. Sci. Paris Sér. I Math. 321 (1995), no. 2, 147-152.

[10] A. V. Gnedbaye: Opérades des algèbres $(k+1)$-aires. Operads: Proceedings of Renaissance Conferences, pages 83-113. Contemp. Math. 202. Amer. Math. Soc., Providence, 1997.

[11] N. Goze, E. Remm: Dimension theorem for free ternary partially associative algebras and applications. J. Algebra 348 (2011) 14-36.

[12] M. MarkL: Models for operads. Comm. Algebra 24, 4 (1996) 1471-1500.

[13] M. Markl, E. Remm: Operads for $n$-ary algebras: calculations and conjectures. Arch. Math. (Brno) 47, 5 (2011) 377-387.

[14] M. MARKL, E. Remm: (Non-)Koszulness of operads for $n$-ary algebras, galgalim and other curiosities. J. Homotopy Relat. Struct. 10, 4 (2015) 939-969.

[15] E. Remm: On the non-Koszulity of the ternary partially associative operad. Proc. Est. Acad. Sci. 59, 4 (2010) 355-363.

Department of Mathematics and Statistics, University of Saskatchewan, Canada

E-mail address: bagherzadeh@math.usask.ca

Department of Mathematics and Statistics, University of Saskatchewan, Canada

E-mail address: bremner@math.usask.ca 